\title{
Dinámicas espaciales recientes de las redes bancarias de España y Brasil: un estudio de las ciudades de Lleida y Presidente Prudente
}

\author{
Ma Pilar Alonso (a), Juliana Oliveira (b), Fabio Fernández (a) \\ (a) Universitat de Lleida, p.alonso@geosoc.udl.cat, fabiofer3103@gmail.com \\ (b) Instituto de Geociências/Universidade Estadual de Campinas, juliana.oliveira@ige.unicamp.br
}

Resumen

En el sistema financiero mundial se han venido produciendo en las últimas décadas importantes transformaciones que han afectado a la presencia espacial de estas actividades. Entre los elementos que han influido más en estos cambios destacan: la mayor interdependencia entre las entidades bancarias por la conformación de un mercado global de capitales; una mayor concentración económica en el sector con intensos procesos de fusiones y adquisiciones bancarias; o la aceleración de las innovaciones tecnológicas, produciendo un incremento del uso de servicios bancarios vía internet. Todo ello lleva a importantes cambios en las lógicas espaciales de los bancos, que tienden a imprimir mayor selectividad en la distribución de sucursales y oficinas bancarias, generando nuevos patrones espaciales en las ciudades. Este trabajo, se centra en identificar cuáles son los rasgos generales y particulares de estos procesos de reconfiguración de las redes bancarias, considerando los cambios a la escala de los territorios nacionales de Brasil y España en el contexto de distintos procesos de crisis económicas, así como la materialización de estos procesos en dos ciudades medias, Lleida (España) y Presidente Prudente (Brasil) entre los años de 2008 a 2016.

Palabras clave: redes bancarias, lógicas espaciales, Lleida, Presidente Prudente.

\section{LAS REDES FINANCIERAS EN EL CAPITALISMO GLOBAL Y SU MATERIALIZACIÓN ESPACIAL}

La fuerte internacionalización e interdependencia de los mercados financieros se manifiesta en diversas escalas y fenómenos económicos, políticos, sociales y también espaciales. La crisis financiera internacional surgida en el verano de 2007 en Estados Unidos, y que se extendió rápidamente a muchos otros territorios en una cascada de caídas financieras, confirmó que el mercado internacional de capitales es uno de los elementos en los que se apoya el capitalismo global (García, F.,2010; Viera, E., 2014).

Los fundamentos de este capitalismo global financiero, fundado en políticas de desregulación del mercado, están basados en un nuevo paradigma técnico que se desarrolla en función de procesos de innovación que facilitan la conformación de un entramado en red, una variable fundamental de la globalización (Minoi, C.; 2012; Santos, M., 2000 Dias, L.C., 2007). La interconexión de las informaciones fundamenta los procesos financieros y se convierte en un elemento fundamental para su desarrollo. Todo ello va unido a una revolución tecnológica profunda, proporcionada por los avances en el progreso técnico de las telecomunicaciones donde la información es la materia prima principal (Castells, M. 2001; Massanel, A., 2016). De este modo "Podemos entonces hablar de una tecnificación del paisaje (...) La información es el vector fundamental del proceso social y los territorios son, de ese modo, equipados para facilitar su circulación" (Santos, M. 2000, p.160 - traducción de los autores).

La conformación de las redes desarrolla nuevos nexos multiescalares entre los territorios - con infraestructuras materiales (los fijos) e tramos inmateriales (los flujos) - configurando un entramado de relaciones económicas, así como nuevas acciones sobre el territorio, que tienen el poder de conectar, desconectar y reconectar los diferentes lugares a esta red global. Las redes financieras globales, a su vez, mantienen su estructura de funcionamiento fundada tanto en los flujos -sobre todo de informaciones- cuanto en las infraestructuras materiales, o fijos, en territorios concretos (sedes de entidades, oficinas, datacenters, cajeros, etc.) con una plasmación local que refleja diferencias y donde los efectos del funcionamiento en red se vuelven más visibles (Carroué, L., 2015).

La conformación de las redes financieras, fuertemente involucradas en la revolución técnica actual (Dabat, A. y otros, 2015), hace emerger en los momentos de crisis económica importantes procesos de reestructuración. La intensidad y las repercusiones de estos procesos vendrán determinados en cada espacio por las debi- 
lidades en la regulación del capital internacional y la fragilidad de los sistemas financieros locales, donde los elementos territoriales visibles (los fijos/redes de oficinas) que apoyan las redes intangibles se ven afectados en diferentes grados y escalas, según las particularidades internas de cada territorio. Son en estas escalas visibles de las redes financieras donde se dejan apreciar las diferencias que la globalización no ha homogeneizado.

Este trabajo, se centra en construir un análisis comparativo sobre las formas de organización de la banca en Brasil y España -señalando procesos generales y particulares que afectaran el sector- así como los cambios espaciales recientes de las redes bancarias en estos países -considerando las dinámicas económicas propias de los dos territorios nacionales-. El objetivo es buscar el marco de explicación de los procesos de reconfiguración espacial bancaria reciente en la escala de dos ciudades medias de los dos países. Los casos de estudio seleccionados son la ciudad de Lleida (España) y la de Presidente Prudente (Brasil) ${ }^{1}$.

La metodología que se sigue para llevar a cabo el análisis planteado se fundamenta en la recopilación, estudio y representación, en forma de tablas, gráficos y mapas, de una serie de variables significativas para observar los cambios en las reconfiguraciones espaciales de las oficinas de la entidades bancarias (evolución del número de entidades financieras y del número de oficinas bancarias, tasas de bancarización, entre otras variables). Los resultados obtenidos se fundamentan en una amplia revisión bibliográfica, que tiene como objetivo ayudar a comprender las particularidades de las distintas formaciones socio-espaciales en estudio, construyendo análisis comparativos entre ellas.

\section{LAS ORGANIZACIONES BANCARIAS EN BRASIL Y EN ESPAÑA. PROCESOS DE REESTRUCTURACIÓN POST-CRISIS}

La globalización ha puesto en marcha redes financieras a escala global, integrando jerárquicamente los mercados y las economías nacionales, cuyo seguimiento material resulta bastante difícil por la rapidez de integración y de movimientos bancarios apoyados en potentes sistemas de información y conexión (Fernández J.C., 2014). Pero junto con los aparentes rasgos de homogeneización de funcionamiento a estas escalas, también hay que valorar las particularidades de estas actividades en distintos contextos territoriales, tratando de entender las materializaciones de las redes de fijos, o sea, las infraestructuras que apoyan su funcionamiento global.

Para comprobar este aspecto, en este trabajo se presenta una caracterización general de la banca en los dos países de los casos de estudio a comparar, buscando identificar rasgos que indiquen semejanzas y diferencias en la forma de organización de sus sistemas bancarios, considerando que ambos tienen también una articulación dentro del mercado financiero globalizado.

Examinando las formas de organización de los sistemas bancarios de Brasil y de España existen similitudes entre los dos países, con presencia de los bancos privados y cooperativas de crédito como intermediarios financieros (ver tabla 1). Aunque también hay diferencias entre los dos países, especialmente con una importante presencia en Brasil de los bancos públicos (los bancos bajo control público con un importante papel social, atendiendo a demandas que los bancos privados no atienden), que demarca una importante particularidad con respecto a

Tabla 1 - Organización del sistema bancario en Brasil y en España. Principales actores.

\begin{tabular}{|c|c|c|}
\hline \multirow{4}{*}{ País } & Tipo de institución & Establecimientos físicos (fijos) \\
\hline \multirow{3}{*}{ BRASIL } & Bancos múltiplos públicos & $\begin{array}{c}\text { Sucursales, Oficinas, Cajeros automáticos, PAB (Puestos de atención bancária) y } \\
\text { "Correspondentes bancários". }\end{array}$ \\
\cline { 2 - 3 } & Bancos múltiplos privados nacionales & $\begin{array}{c}\text { Sucursales, Oficinas, Cajeros automáticos, PAB (Puestos de atención bancária) y } \\
\text { "Correspondentes bancários". }\end{array}$ \\
\cline { 2 - 3 } & Bancos múltiplos privados internacionales & $\begin{array}{c}\text { Sucursales, Oficinas, Cajeros automáticos, PAB (Puestos de atención bancária) y } \\
\text { "Correspondentes bancários". }\end{array}$ \\
\cline { 2 - 3 } & Cooperativas de crédito & Sucursales y Oficinas \\
\cline { 2 - 3 } ESPAÑ & Banca tradicional & Sedes, oficinas y Cajeros automáticos \\
\cline { 2 - 3 } & Cooperativas de crédito & Sedes, oficinas y Cajeros automáticos \\
\cline { 2 - 3 } & Cajas de ahorro & Sedes, oficinas y Cajeros automáticos \\
\hline
\end{tabular}

Fuente: Elaboración propia, 2018

\footnotetext{
${ }^{1}$ Los resultados presentados en este trabajo son fruto de una cooperación entre la Universitat de Lleida y la Universidade Estadual Paulista, con beca financiada por la Fundação de Amparo à Pesquisa do Estado de São Paulo (Fapesp).
} 
España donde no existe este tipo de instituciones. Por otro lado en España, la presencia de las cajas de ahorro durante mucho tiempo expresaron una particularidad de su sistema bancario. Estas instituciones, a pesar de ser privadas, también tenían un importante papel social y de financiación de familias y pequeñas empresas, componiendo una amplia red de talleres sobre el territorio (Alonso, M.P. y otros, 2014 a).

Además de los aspectos relacionados a los actores que actúan en el sector bancario en los dos países, también identificamos semejanzas y diferencias en los tipos de establecimientos que componen las capacidades instaladas del sector sobre el territorio, que estructuran su red física de gestión e intermediación financiera. En los dos países hay presencia de sucursales/sedes de las instituciones, oficinas bancarias y cajeros automáticos como los fijos fundamentales para el funcionamiento del sistema bancario, y cuyas lógicas de localización son muy similares, pero también hay otras que presentan lógicas espaciales propias que componen la topología del sector en cada país (Contel, F., 2009), como se resume en la tabla 2.

Tabla 2 - Tipos de establecimientos bancarios, funciones y lógicas espaciales.

\begin{tabular}{|c|c|c|}
\hline $\begin{array}{c}\text { Tipo de establecimiento } \\
\text { (Fijo) }\end{array}$ & Funciones & Lógicas espaciales \\
\hline Sucursales/Sedes & $\begin{array}{c}\text { Centraliza la gestión de las actividades administrativas } \\
\text { y financieras de la institución bancaria. }\end{array}$ & $\begin{array}{c}\text { Localización centralizada en ejes especializados, sobre } \\
\text { todo en los centros económicos más importantes del } \\
\text { país. }\end{array}$ \\
\hline Oficinas & $\begin{array}{c}\text { Ofrecen todos los servicios de intermediación finan- } \\
\text { ciera para sus clientes. }\end{array}$ & $\begin{array}{c}\text { Localización más dispersa, pero que privilegia las áreas } \\
\text { centrales con accesibilidad a gran número de perso- } \\
\text { nas. En Brasil, se observa una orientación a la instala- } \\
\text { ción de esos establecimientos en el centro tradicional } \\
\text { y en las áreas de las ciudades donde residen poblacio- } \\
\text { nes de mayor poder adquisitivo. En España también } \\
\text { existe una tendencia a la centralización. }\end{array}$ \\
\hline PAB - Puesto de atención \\
bancária (Brasil) & $\begin{array}{c}\text { Ofrece servicios más específicos y limitados de inter- } \\
\text { mediación financiera para un público específico } \\
\text { (funcionarios, estudiantes, etc.). }\end{array}$ & $\begin{array}{c}\text { Se sitúan lo más cerca posible a los lugares más acce- } \\
\text { sibles a su público: administración, universidades, } \\
\text { centros empresariales, etc. }\end{array}$ \\
\hline "Correspondentes bancá- \\
rios" (Brasil) & $\begin{array}{c}\text { Son establecimientos que actúan ofreciendo algunos } \\
\text { servicios bancarios básicos (jubilaciones, pago de } \\
\text { cuentas, transferencias, etc.). Estos establecimientos } \\
\text { actúan bajo la supervisión de los bancos, sin embargo } \\
\text { de manera externalizada. }\end{array}$ & $\begin{array}{c}\text { Se caracterizan por un mayor acercamiento a los } \\
\text { clientes, aprovechándose de la ubicación central de } \\
\text { establecimientos comerciales de barrios, como merca- } \\
\text { dos, panaderías, farmacias, etc. }\end{array}$ \\
\hline Cajeros Automáticos & $\begin{array}{c}\text { Son equipos electrónicos bancarios, que ofrecen auto- } \\
\text { servicio para realizar algunas transacciones básicas }\end{array}$ & $\begin{array}{c}\text { Ubstratégicos y accesibles a gran número de personas, } \\
\text { como gasolineras, centros comerciales, mercados, etc. }\end{array}$ \\
\hline
\end{tabular}

Fuente: Elaboración propia, 2018.

En el caso de Brasil las dos particularidades más significativas, en relación a los tipos de establecimiento, se refieren la primera a la presencia de los $\mathrm{PAB}$, que son puestos de atención orientados a públicos específicos y ubicados en locales muy accesibles (edificios de administraciones públicas, centros industriales y empresariales, así como en empresas con un gran número de trabajadores), actúan de manera similar a las oficinas, pero ofreciendo servicios más básicos, operaciones más limitadas; el segundo son los "correspondentes bancarios" actúan como intermediarios financieros sobre la supervisión de los bancos tradicionales, ubicados en lugares de paso, cuyas lógicas espaciales están dirigidas a la mayor proximidad a los clientes, sobre todo en áreas no atendidas por establecimientos bancarios como oficinas y cajeros, generando una ampliación de la red de servicios bancarios con una menor inversión del sector, pueden estar instalados en establecimientos como supermercados, oficinas de correos, farmacias, entre otros (Contel, 2011). Tienen una lógica de mayor acercamiento a los clientes.

En el caso español, cabe destacar el papel particular de las cajas de ahorro, que tenían un reparto muy local y próximo a los territorios, con una fuerte dispersión espacial de oficinas por las ciudades y las áreas rurales, por lo tanto, con gran importancia en la composición de las redes bancarias en España. Sin embargo, con el tiempo este tipo de entidades también se fueron involucrando en las inversiones de sus administraciones regionales públicas, y poco a poco cambiando su filosofía hacia la de la banca tradicional y entrando a formar parte de las redes de financiación internacional, lo que llevo con la crisis y los problemas de las inversiones inmobiliarias a su casi desaparición en el contexto post-crisis. El resultado ha sido un refuerzo de la centralización de sedes bancarias (con una ubicación central en todas las escalas) y una menor presencia de oficinas en el territorio tras un fuerte proceso de cierres y una búsqueda también de la centralidad espacial.

Brasil y España parten, por tanto, de algunas diferencias en las tipologías de entidades en su redes bancarias internas aún que sometidas a procesos globales cada vez se asemejan más. Así, hay que indicar que en los últimos 
años, en ambos países, se han vuelto cada vez más importante las funciones de auto-atención al cliente por canales remotos, destacando el uso la banca online, que ha crecido en número de usuarios y en volúmenes de transacciones, afectando los ritmos de expansión de las redes de atención presencial en estos territorios.

Por otra parte, al analizar la trayectoria del número de entidades actuales en los dos países se aprecian procesos parecidos de concentración bancaria en el sector, sobre todo si se consideran los bancos tradicionales en Brasil, y las cajas de ahorro en España. Aunque el estudio de las trayectorias reflejan en ambos un número cada vez más reducido de entidades que lleva a su concentración espacial.

En Brasil, en la década de 1990, actuaban cerca de 240 entidades bancarias (bancos tradicionales), pasando a 157 en el año 2016, tras dos décadas marcadas por importantes procesos de concentración en el sector, sobre todo con la entrada de inversiones internacionales en la economía brasileña a lo largo de los años 90 e inicio de los años 2000 (Días, L., 2009; Contel, F., 2011; Banco Central do Brasil, 2016). En España, en la década de 1990 actuaban 154 entidades bancarias (bancos tradicionales) y 64 cajas de ahorro, ya en el año 2016 se registraron 130 bancos en funcionamiento y solamente 2 cajas de ahorro en funcionamiento (Anuario Banco de España, 2016; Anuario CECA, 2016), fruto de procesos de fusiones y adquisiciones bancarias en función de la reestructuración post-crisis (Ver gráfico 1).

Grafico 1 - Evolución del número de entidades bancarias en Brasil y España, 1990 a 2016.

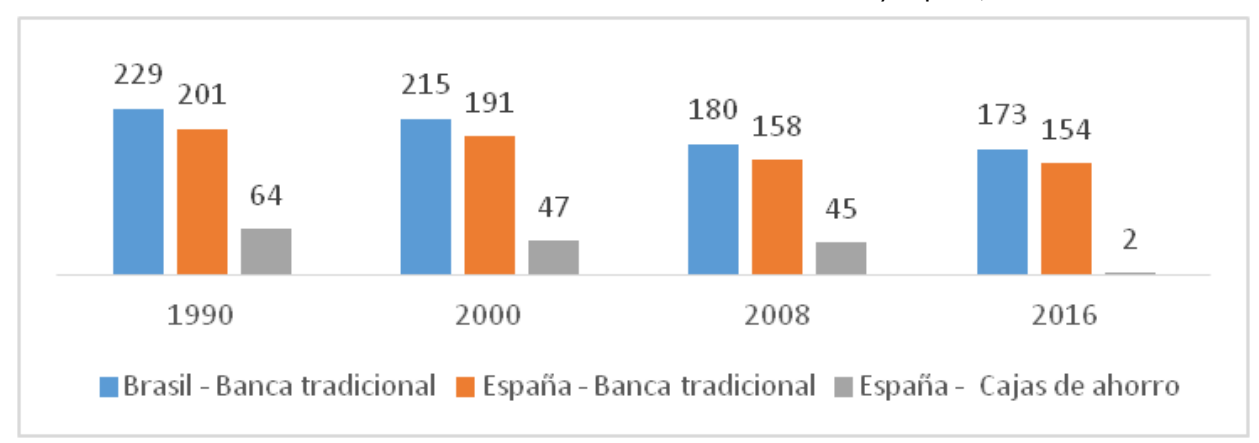

Fuente: elaboración propia a partir de datos del Banco Central do Brasil, 2016, Anuario Banco de España, 2016 y Anuario CECA, 2016

Si se compara la trayectoria de las tasas de bancarización de ambos países se puede observar como, a pesar de los diferentes contextos económicos que registran España y Brasil, ambos países muestran una trayectoria positiva en el número de cuentas bancarias activas en bancos tradicionales ${ }^{2}$, tendencia que se articula a las crecientes capacidades de expansión de las finanzas como variable presente y necesaria en el funcionamiento económico actual y marcada por la hegemonía del mercado globalizado.

En Brasil este crecimiento fue más acelerado en los últimos años, sobre todo a causa de las políticas de inclusión social y el aumento de las tasas de empleo, lo que aumentó el porcentaje de población con acceso a poseer una cuenta bancaria. España, por su parte, también presentó un crecimiento en el número de cuentas bancarias, aunque en menor ritmo que Brasil. A pesar de ello, España presenta mayores tasas de bancarización, es decir, una parte mayor de la población española está incluida en el sistema financiero en comparación con Brasil. En cualquier caso la tendencia de bancarización es creciente en los dos países (Ver gráfico 2).

A pesar de la tendencia de mayor bancarización en cuanto a cuentas abiertas, se puede observar que en los dos países estudiados las redes presenciales de oficinas bancarias ha disminuido su presencia de manera importante - sobre todo en España - fruto del contexto de reestructuración bancaria sufrido, aunque lo han hecho de diferentes maneras y en distintos períodos. Así, en España los cambios económicos generados a raíz de la crisis internacional de 2007 marca el inicio del proceso de reestructuración en su sistema bancario, proceso que dura hasta el presente. Durante los primeros años del S XXI las entidades financieras apostaron por una fuerte concesión de créditos hipotecarios que generó una dinámica insostenible para muchas entidades por su fuerte endeudamiento y que se destapó con la crisis internacional (Alonso, M.P. y otros, 2014 a). Con el estallido de una enorme burbuja inmobiliaria fue necesario un reajuste del modelo financiero que rebota directamente sobre la reestructuración de todo el sistema bancario (Ver gráfico 3), con la reducción de entidades financieras (fusiones, ad-

\footnotetext{
${ }^{2}$ El número de cuentas bancarias se refiere al total de cuentas corrientes y cuentas de ahorro activas en la banca tradicional.
} 
sorciones), de las oficinas de atención al cliente y de los puestos de trabajo del sector (Alonso, M.P. y otros, 2014 a; Mateache, P., 2012).

Gráfico 2 - Número total de cuentas bancarias activas y tasa porcentual de bancarización ${ }^{3}$ en la banca tradicional de Brasil y España, 2008-2016.

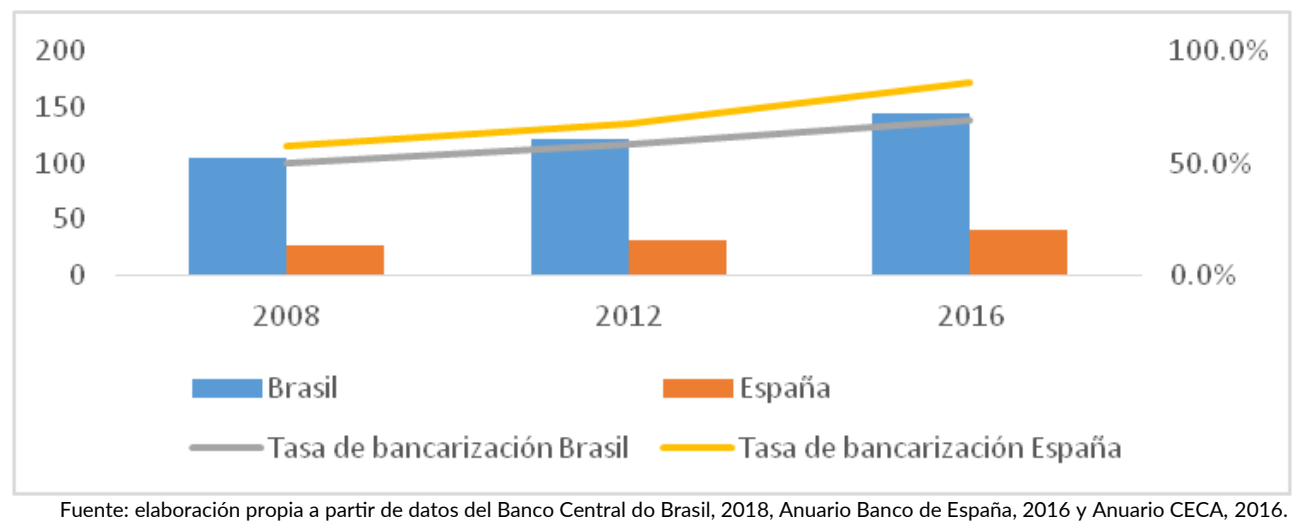

Gráfico 3 - España. Evolución del número de oficinas de bancos tradicionales y cajas de ahorro entre 2000 y 2016.

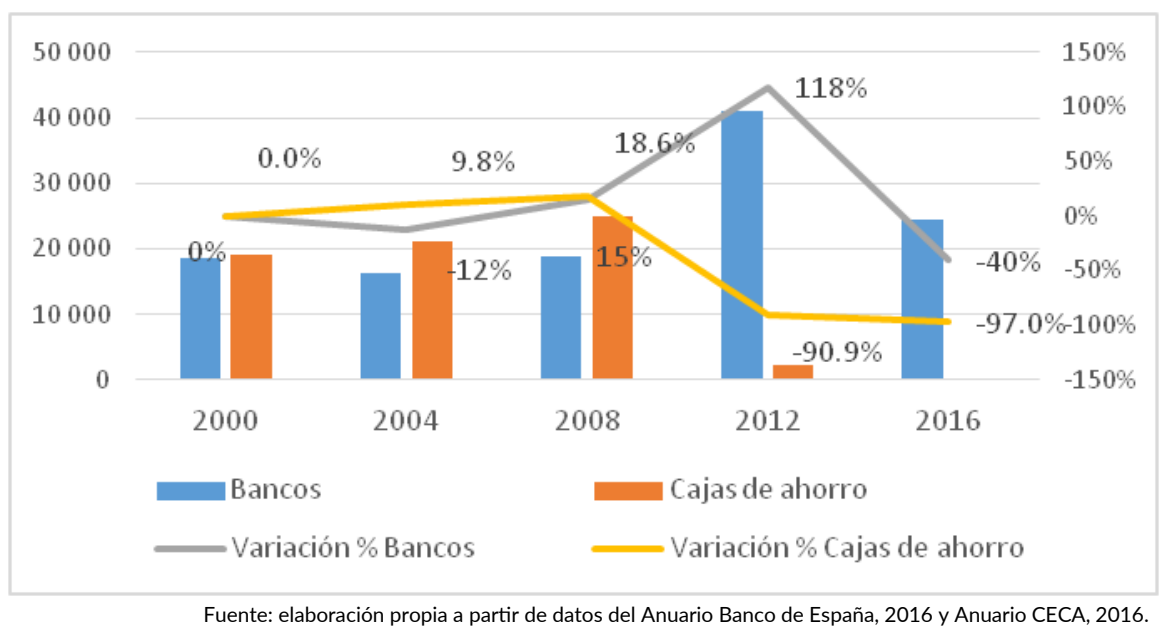

En Brasil los cambios que han afectado a las redes bancarias empezaran más tarde, con la crisis económica y política que estalla en el año 2015 (crisis de precios de las commodities, desaceleración del crecimiento económico, disminución de la recaudación fiscal, implantación de políticas de austeridad fiscal,...), procesos que suponen el inicio de su actual reestructuración bancaria, sobre todo por el intento de reducción de costos operativos del sector y también por el aumento de las inversiones en otros canales de autoservicio vía internet, generando un cambio en la forma de ofrecimiento de servicios bancarios. La consecuencia más visible en el territorio también está siendo la reducción del ritmo de expansión de las entidades y oficinas bancarias (Ver gráfico 4) fundamentada en el discurso de los bancos - sobre todo de los bancos públicos - de reducción de los costos operativos por medio de disminución de las redes de atención presencial, principalmente en las áreas dotadas con mayor número de oficinas, como medio de garantizar liquidez, empezando, así, cambios basados en la disminución de la capacidad instalada (Oliveira, J., 2017).

Por tanto, ambos países, en distintos momentos, sufren una reconfiguración que lleva a esa reducción de entidades y de oficinas, fruto de diferentes procesos de crisis. Estas situaciones han generado efectos particulares sobre las formas de organización de la banca y sobre la distribución y expansión de las redes de atención presencial.

Frente a esto en estos momento ambos países están siendo afectados por las modernizaciones tecnológicas

${ }^{3}$ La tasa de bancarización se calculó con base en la relación porcentual entre el número de cuentas corrientes y cuentas de ahorro y total de población de ambos países. 
Gráfico 4 - Brasil. Evolución del número de oficinas bancarias y variación porcentual entre 1990 y 2016.

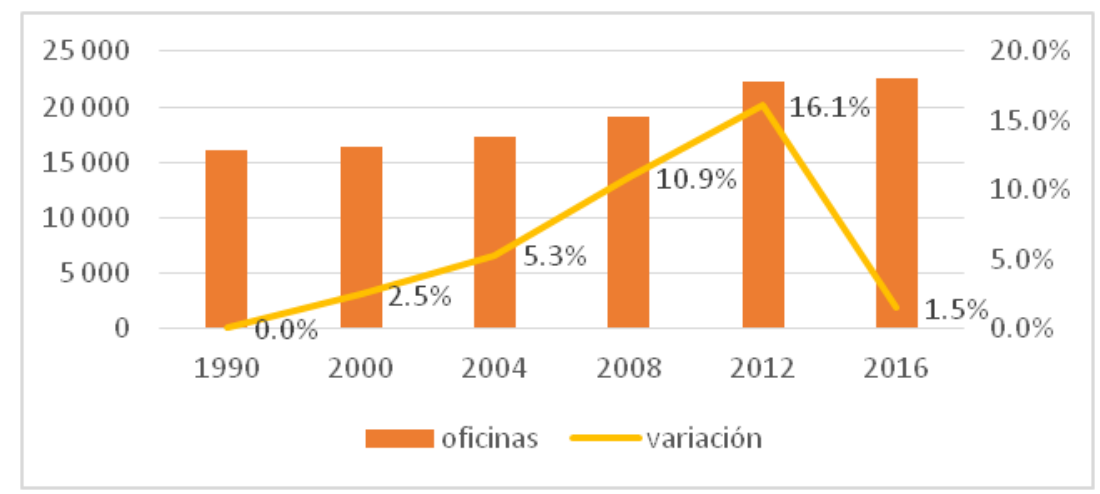

Fuente: elaboración propia a partir de datos del Banco Central do Brasil, 2018.

en el sector bancario de cara al cliente, que repercuten fuertemente en los dos países observados ${ }^{4}$. Los resultados se traducen en la diseminación del uso de servicios digitales, proporcionando un refuerzo de la reducción en el número de oficinas, dada la posibilidad de expansión del autoservicio del cliente, con máxima reducción de los costos por transacción, haciendo reducir la importancia de la atención presencial (Febraban, 2015; Massanell, A., 2016).

En definitiva, la caracterización realizada de la situación de la banca en Brasil y España confirma que sus contextos de configuración de las entidades bancarias han sido distintos y con cronologías de desarrollo diferentes, pero donde parece que para la prestación de servicios on line sí que está generando efectos similares: una importante reducción de las redes de oficinas bancarias en los últimos años, generando una reestructuración espacial, aún en curso, en el sector bancario.

\section{LÓGICAS ESPACIALES DE LA BANCA EN LAS CIUDADES DE PRESIDENTE PRUDENTE Y LLEIDA}

Los procesos particulares de reestructuración de las redes bancarias en Brasil y España -fundados tanto en un contexto de crisis como en una fuerte transformación en el modelo de ofrecimiento de servicios bancarios en razón de las innovaciones en el sector- marcados sobre todo por la disminución de capacidad instalada, repercutirán en nuevas situaciones espaciales de las oficinas en sus ciudades. Se han modificado los modelos de expansión de las redes bancarias así como sus lógicas espaciales y afectando a los hábitos de los clientes. Para una mayor compresión de estos procesos se presenta un acercamiento a lo acaecido sobre dos ciudades medias, una de Brasil -Presidente Prudente- y otra España -Lleida-.

La selección de esta tipología de ciudades medias está relacionada con el importante papel que juegan en intermediación como centros de referencia para un territorio próximo con muy poca presencia de los servicios bancarios, sobre todo en el caso de Brasil, donde la atracción de los servicios bancarios en las ciudades es más importante (Sposito, M.E., 2007:1983; Oliveira, J., 2015; Bellet, C., y otros, 2015). Por este motivo, la selección de las ciudades de Lleida y Presidente Prudente, permiten mostrar los resultados de la reestructuración del sector bancario de una manera más concreta, y donde al igual que otra tipología de ciudades también se ha perdido capacidad de oficinas presenciales (Alonso, M. P. y otros, 2014 b)

Lleida es una capital provincial ubicada en el interior de la comunidad autónoma de Cataluña en el noreste de España. Cuenta con una población de 137.327 habitantes (Idescat, 2017), una superficie de 212,3 Km² y una densidad demográfica de $646,9 \mathrm{hab} / \mathrm{km}^{2}$. En ella, como en otras ciudades de esta categoría, se habían concentrado un número importante de entidades y de oficinas bancarias que acercaban al cliente a estos servicios, desarroIlando un relevante papel de intermediación financiera (Fernández, F., 2014). Proceso similar a muchas otras ciudades españolas, que durante años vieron como la implantación bancaria llegaba a todos los ciudadanos, generando ratios de oficina-cliente muy bajos (Alonso, y otros, 2014, a).

${ }^{4}$ En España según Statista en 2006, el número de usuarios de la banca on line era del 15\%, en 2017 de 46\%, en Brasil, según datos de Febraban (2017), el número de cuentas bancárias con acesso a la banca on line evoluiu de 27 millones, en 2016, a 53 millones en 2016 
La ciudad de Presidente Prudente se ubica en el interior del estado de São Paulo. Su población es de 225.271 habitantes (IBGE, 2017), su superficie de $562,107 \mathrm{~km}^{2}$ y su densidad demográfica de $368,89 \mathrm{hab} / \mathrm{km}^{2}$. Es sede de la mesorregión y microrregión de mismo nombre y también desarrolla importantes funciones de intermediación urbana y financiera (Sposito, M.E., 1983; 2007). Esta ciudad ejemplifica el comportamiento de muchas otras ciudades brasileñas donde no toda la población ha tenido un acceso fácil a disponer de cuentas bancarias y por tanto de necesidades de entidades. Esta situación ha tenido una repercusión espacial, con centralidad hacia la población que tenía capacidad de disponer de cuentas bancarias.

Teniendo en cuenta las características fundamentales de estas ciudades y los contextos económicos y organizativos de las estructuras bancarias de sus países, presentados en lo apartado anterior, el objetivo de este punto es construir un análisis que permita establecer paralelismos entre las lógicas espaciales del sistema bancario en dos ciudades tan distantes y ver si los procesos de globalización financiera en las escalas locales dejan las mismas plasmaciones espaciales en ellas. Para ello se examinan las siguientes variables: trayectorias de evolución del número de oficinas bancarias y lógicas espaciales adoptadas por el sector a partir de las distintas coyunturas de crisis definidas en los dos países a las que pertenecen.

En relación a la trayectoria reciente del número de oficinas bancarias en las dos ciudades, es posible observar que en Lleida (Ver gráfico 5), a partir de 2008, la capacidad instalada ha ido disminuyendo de forma notable, siendo 2012-2013 los años que registraron la mayor caída (-13,7\%). Sin embargo en la post-crisis los cierres han continuado y el balance a finales de 2016 es que de 2008 a 2016 en la ciudad de Lleida han cerrado un total de 97 oficinas es decir casi el $50 \%$ de las que había al inicio de la crisis.

Esta evolución negativa de la trayectoria del número de oficinas bancarias en la ciudad de Lleida se relaciona en su origen, como se ha comentado, con la reestructuración del sector debido a una serie de normativas de la banca española que actuaban en el sentido de un reajuste del modelo financiero y que rebota directamente sobre la reestructuración de todo el sistema bancario español a partir de $2009^{5}$. El resultado fue un endurecimiento de la regulación en el año 2011, generando una aceleración de los cierres y produciendo efectos espaciales en el estudio de caso, Lleida, y en muchas otras ciudades españolas. Se produce una fuerte disminución del número de oficinas y entidades, y como resultado se aprecia como muchos barrios de Lleida se quedan casi sin servicios financieros o como en los espacios centrales también ven reducida la presencia de entidades y oficinas (Fernández, F., 2014).

En los últimos años, Lleida ha seguido perdiendo oficinas, pero en este caso la reestructuración se asocia, como se ha comentado, más a la propia reorganización de las oficinas ante el incremento de la potenciación de la banca on line por parte de las distintas entidades bancarias. En toda la banca española se están reforzando los cierres en razón de la disminución de costos proporcionada por estos nuevos canales de realización de transacciones bancarias fundadas en el autoservicio y en lo digital (Troiano, M., y otros, 2014; Garrido, A., 2017). La consecuencia más visible es el claro recorte de la capacidad instalada, y un descenso el número de empleados que se dedican a la atención presencial al cliente y también en la presencia de la red física de oficinas.

En cuanto a la evolución de las oficinas en la ciudad de Presidente Prudente (Ver gráfico 5), se aprecian importantes diferencias en la cronología con la trayectoria experimentada en Lleida en el periodo examinado. Así entre 2008 y 2011 en Presidente Prudente se registraron pequeños incrementos de oficinas (3\% y $8 \%$ ), eso se debe, como se ha comentado, por el contexto económico de expansión por el cual atravesaba Brasil, conformando condiciones favorables a la expansión de las redes bancarias, aunque en volúmenes por debajo de lo necesario, con un importante proceso de concentración en los lugares centrales. Sin embargo entre los años 2011 y 2013 ocurren pequeñas caídas en los números totales de fijos, respectivamente de $-2 \%$ y $-1 \%$. Esta tendencia se refuerza a partir de 2014, con caídas consecutivas de $-6 \%$ y $-27 \%$ en los años de 2015 y 2016 , fruto de los efectos de la situación de crisis económica desencadenada en Brasil en el año 2015 que, a su vez, reforzó el discurso de necesidad de reducción de la capacidad instalada de los bancos a fin de garantizar la liquidez en esos momentos de crisis. Pero a diferencia de lo ocurrido en las ciudades españolas, donde tras un fuerte ajuste físico del número de entidades y de oficinas, la aceleración en el uso de las TIC fomenta una nueva perdida de la capacidad presencial (Maudos, 2014), en el caso de Brasil se produce todo en paralelo, la reestructuración del sector para adaptarse a la crisis del país y a la vez se está desarrollando con un modelo fundado en la banca digital, sobre todo a partir de 2014, con fuertes inversiones en TIC, de manera que este nuevo modelo repercute también en la disminución del número de oficinas bancarias, privilegiando las plataformas online para ofrecer sus servicios

\footnotetext{
${ }^{8}$ Para Brasil se consideraron las oficinas de bancos tradicionales (oficinas y PAB) y cooperativas de crédito (oficinas); Para Lleida se consideraron las oficinas de bancos tradicionales, cooperativas y cajas de ahorro.

${ }^{5}$ Fondo de Reestructuración Ordenada Bancaria (FROB), establecida en septiembre de 2009.
} 
(Oliveira, J., 2017). Así, la "Federação Brasileira de Bancos" afirma que los puntos más relevantes para entender la diminución de las redes de atención presencial son:

(...) a conjuntura econômica vivida pelo País, que culminou no fechamento de uma série de estabelecimentos; a política de eficiência operacional de alguns bancos, que optaram em realizar uma revisão para solucionar sobreposições de pontos de atendimento existentes; os movimentos de consolidação no mercado por meio de fusões e aquisições; e a própria diversificação dos canais de atendimento (Febraban, 2015, p.27).

Por tanto, es necesario apuntar que las reestructuraciones más recientes sufridas por las entidades financieras en los espacios urbanos están ligadas sobre todo con la renovada ola de innovación en el sector bancario (Cernev, A. et. al., 2009; Oliveira, J., 2017; Carbó, S., 2017), asociada al actual momento de desarrollo técnico a nivel global (Castells, M., 2001; Massanell, A., 2016), y que genera nuevas variables que contribuyen a cambios en el patrón de expansión de los fijos bancarios (red de oficinas) a nivel global, incluso figurando como un rasgo común entre Brasil y España, reflejando un proceso de homogenización técnica propio de la globalización (Santos, M., 2000; Castells, M., 2001; Harvey, D., 1993), aún que repercutiendo de forma particular en los diferentes países. Al creciente uso de los canales digitales contribuyen los nuevos patrones de demanda social, cada vez más implicada en los procesos digitales, así como la presencia de nuevos competidores muy especializados en determinados servicios financieros como las Fintech (empresas apoyadas en nuevas tecnologías para ofrecer servicios financieros), o las firmas de comercio electrónico y telefonía (Amazón, Alibaba, Samsung Pay, Apple Pay, BBV, NFC Movistar, Vodafone, Wallet, etc.) (Rojas, L., 2016), las entidades financieras ven que estas nuevas formas de funcionamiento abaratan costes y por tanto están buscando formas combinadas de funcionamiento.

Gráfico 5 - Evolución del número de oficinas bancarias en Presidente Prudente y Lleida (2008-16).

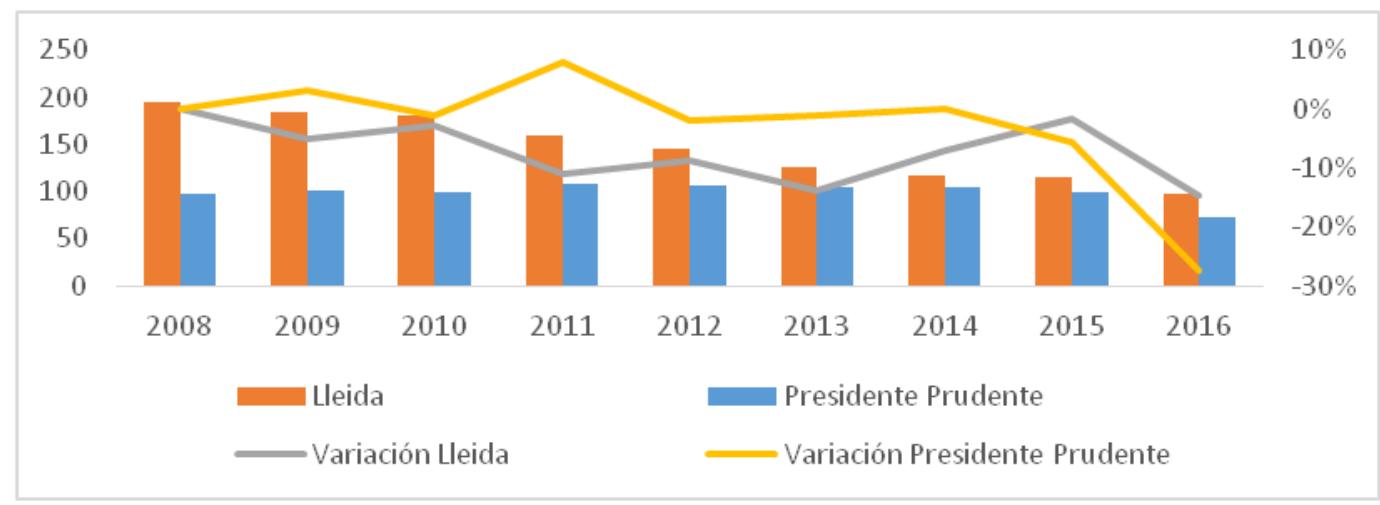

Fuente: Elaboración propia a partir de trabajo de campo y consulta de las webs.

Pero la menor presencia de oficinas de gestión bancaria supone problemas importantes que llevan hacia procesos de exclusión. Hay grupos sociales que todavía dependen mucho de los servicios bancarios presenciales, sobre todo la población de más edad, de menores ingresos y los excluidos digitales, en este sentido, a pesar de la creciente relevancia de los servicios digitales a través de Internet, las redes de atención presenciales siguen siendo parte esencial para la realización de las actividades bancarias cotidianas para una parte muy importante de la población (Garrocho, C.f. y Campos, J., 2010; Oliveira, J., 2017).

En lo que respecta a las lógicas espaciales de este sector en ambas realidades urbanas observadas, se pueden apreciar algunas diferencias originadas por su configuración inicial, pero que con las últimas transformaciones que van sufriendo tienden a asemejarlas. Así, en Presidente Prudente históricamente había una mayor concentración de oficinas en el sector sur de la ciudad, principalmente en el centro tradicional y en algunos otros ejes viarios y comerciales de mayor accesibilidad (Mapa 1) (Oliveira, J., 2015). Con la continua expansión del tejido urbano de la ciudad el sector bancario también llevó a cabo una expansión de las oficinas, aunque se centró en las áreas donde residía la población con mayor poder adquisitivo y no cubriendo a las zonas residenciales con menor poder adquisitivo - sector norte -. Esta situación ha venido reforzando la exclusión financiera en la ciudad, la población que vive más alejada del centro debe realizar mayores desplazamientos para acceder a servicios bancarios (Oliveira, J., 2015), además hay que valorar que se trata de una estructura urbana dispersa en sus bordes que posee baja densidad poblacional, lo que dificulta medios de transporte colectivos. Como se puede observar en la 
cartografía de localización de oficinas (Ver mapa 1), se aprecia la centralidad de oficinas y observando el mapa de cierres, donde los puntos rojos que indican las oficinas cerradas en el período 2010-2015, se detecta que se han perdido oficinas en el área central de la ciudad, pero también se han cerrado establecimientos en barrios ya poco provistos de esos fijos, y donde el problema de exclusión se acentúa más.

En cuanto a la organización del sector bancario en la ciudad de Lleida, se dispone de información de localización para los años de 2010-2015 y de su variación para ver los cambios que se aprecian (Ver mapa 2). Así en 2010 se puede ver una mayor presencia de oficinas por toda la ciudad y, por lo tanto, una oferta más amplia de servicios con presencia de establecimientos en prácticamente todos los barrios y de manera más numerosa en los sectores centrales. Sin embargo, los procesos de cierres de oficinas causados por la reestructuración del sector han hecho disminuir la densidad de la red de oficinas, consolidando una estructura espacial bancaria más central que va dejando sin acceso fácil de estos servicios a las personas que viven en zonas alejadas, comparativamente acercándose a la situación de centralidad de estos servicios que presenta la ciudad de Presidente Prudente (Ver mapas 1 y 2).

Mapa 1 - Distribución de oficinas bancarias e localización de las oficinas cerradas en Presidente Prudente, 2010-2015.
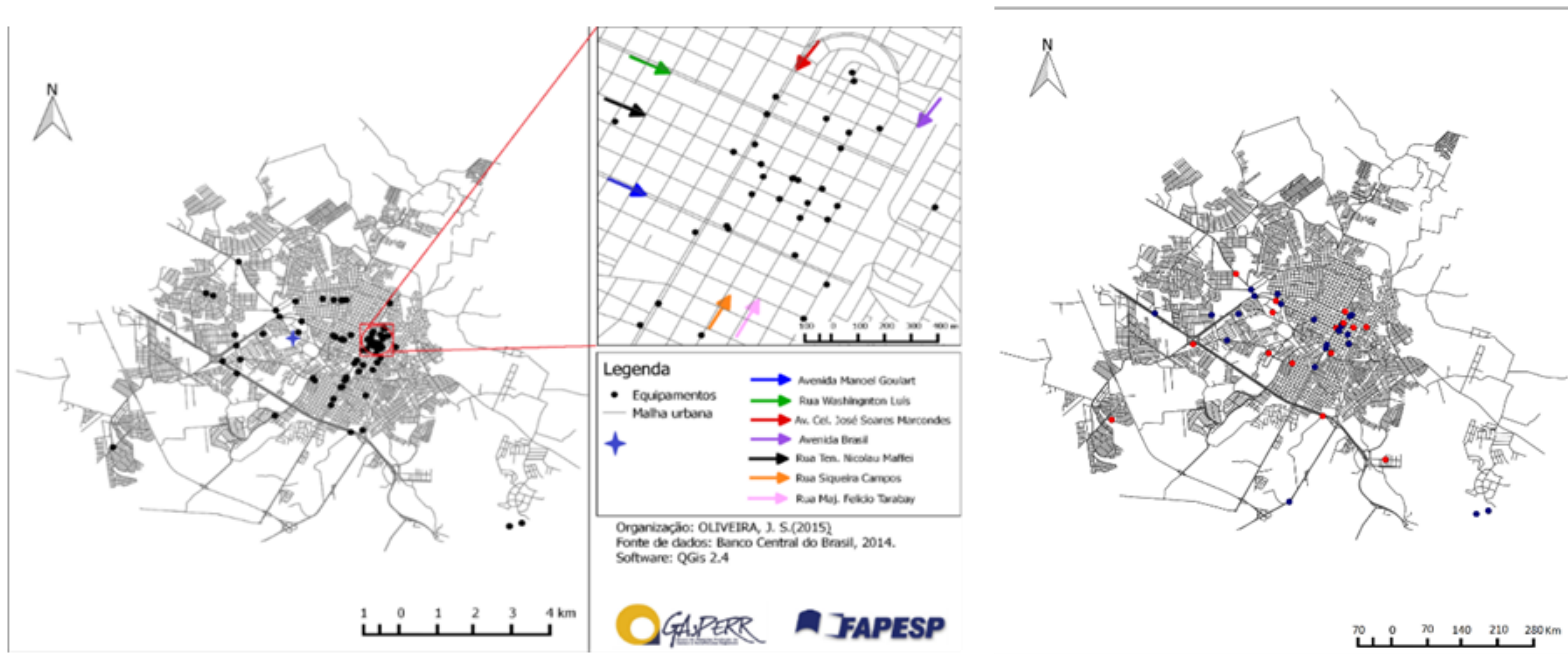

Fuente: Elaboración propia a partir de los datos del Banco Central do Brasil, 2014

Mapa 2 - Distribución de oficinas bancarias en Lleida, 2010-15 y cierres.

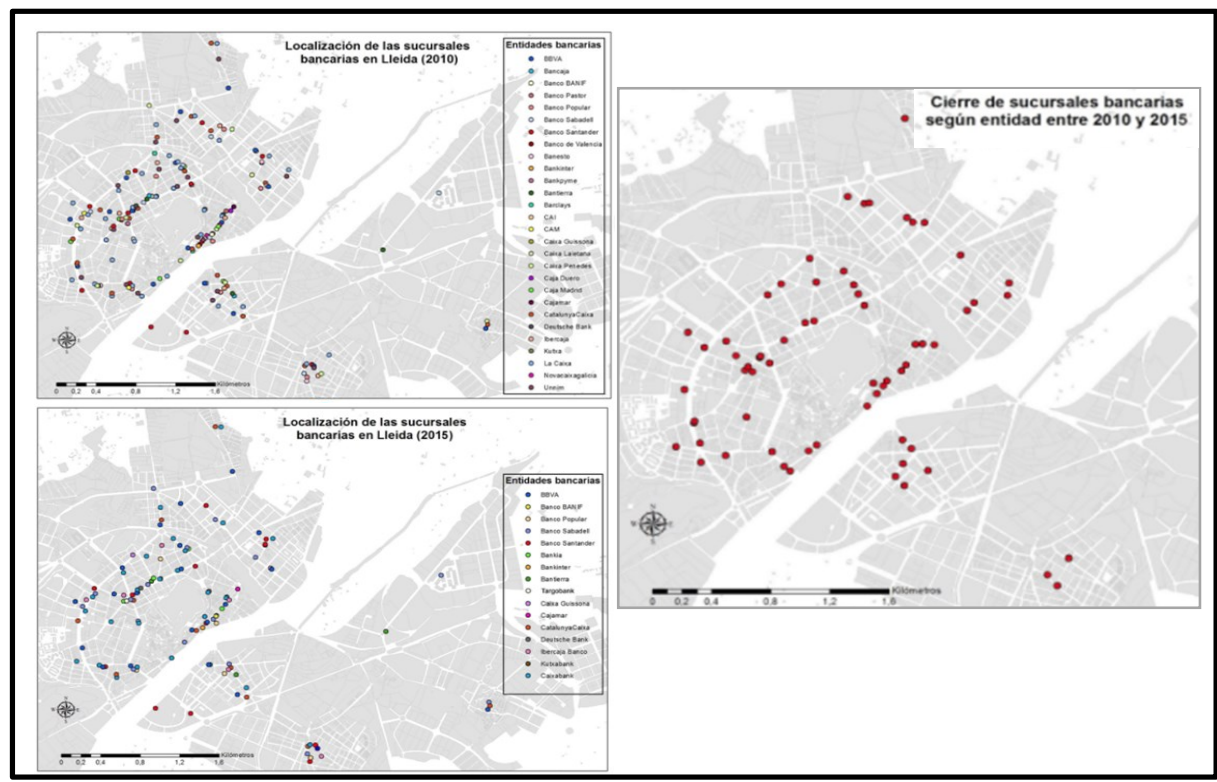

Fuente: Elaboración propia a partir de trabajo de campo y páginas web de sucursales bancarias. 
En resumen, a pesar de que las dos ciudades van a pasar por procesos de reorganización de sus localizaciones de establecimientos bancarios, sus formas de actuar han sido distintas, sobre todo porque partían de tipologías de implantación diferentes. En el caso de Lleida se había tendido hacía un modelo de acercamiento de las oficinas a los clientes, aunque en 2016 la situación era muy distinta, en concreto más de 98 oficinas perdidas, y un refuerzo de la centralidad en las que van quedando. En el caso de Presidente Prudente, ha predominado la centralidad de la función desde el principio, aunque con un período de una relativa difusión selectiva de oficinas que reforzó las desigualdades de acceso a estos servicios, acercándose hacia zonas con clientes potenciales y no hacía las áreas donde residen poblaciones menos favorecidas.

Lo que sí ambas ciudades están llevando más en paralelo es el uso de la banca digital, que también se traduce en cierres de oficinas presenciales, reforzando un modelo concentrado de la banca con presencia física. Estas tendencias de reducción de las capacidades instaladas, imponen un riesgo para el desarrollo pleno de esos territorios, ya que, en un contexto global en que las finanzas son los motores de los territorios es evidente la necesidad de que los lugares sean dotados de oficinas físicas y redes bancarias que propicien capacidades de intermediación financiera (Scherma, R., 2008, Maudos, 2014).

\section{CONCLUSIONES}

Las transformaciones que se desarrollan a nivel global en el funcionamiento del sistema financiero tiene su plasmación en los cambios recientes en la organización de la banca en Brasil y España, pero los procesos de cambios han estado asociados a las particularidades tradicionales de los diferentes territorios. De este modo, los procesos de concentración económica en el sector bancario, así como los recientes cambios tecnológicos incorporados a su estructura de funcionamiento, en ambos países están generando un nuevo modelo de atención bancaria, que repercuten en la reducción de las capacidades instaladas de las redes bancarias en los dos países, acentuándose en los en los últimos años.

Pero en este contexto de cambios hay que valorar que las dinámicas de reestructuración de las redes bancarias en Brasil y en España no se procesaran en el mismo contexto, tanto desde el punto de vista temporal -la reestructuración bancaria en España ocurre en el contexto de la Gran Recesión, mientras que la reestructuración bancaria en Brasil es más reciente, a partir de 2015-, como desde el punto de vista de los diferentes niveles de impacto en las redes de oficinas -siendo en España la reconfiguración de estas redes es bastante más profunda que en Brasil, cuyo proceso ocurre de forma más gradual y concentrada en las áreas con mayores densidades de oficinas -.

Las repercusiones de las reestructuraciones en el sector se materializan de forma más visibles en las escalas locales, así en el análisis realizado sobre las dos ciudades medias, se ha podido observar que se han generado importantes cambios en el número disponible de oficinas bancarias, así como en las lógicas espaciales de estas redes. En Lleida los distintos procesos globales acaecidos ha hecho disminuir el número de oficinas en toda la ciudad, pero sobre todo en los barrios, concentrándose de forma más marcada en el centro tradicional, con menor accesibilidad a las poblaciones más alejadas. En Presidente Prudente los cierres producidos refuerzan una estructura espacial históricamente desigual en la distribución de estos fijos, con fuerte concentración de oficinas en el centro tradicional y en las áreas donde residen las poblaciones de mayores ingresos. Con los cierres recientes de oficinas en las áreas centrales y algunos barrios, y sin perspectivas futuras de expansión de esas redes hasta las áreas donde residen las poblaciones más pobres, la tendencia de exclusión a ciertos servicios bancarios se intensifica en esta ciudad.

Tanto en Lleida como en Presidente Prudente, las nuevas lógicas de localización de las redes de oficinas, con disminución de la presencia en espacios periféricos, están exigiendo mayores desplazamientos para acceder a estos servicios de marera presencial, lo que supone procesos de exclusión hacia ciertos grupos de la población como las personas de más edad, los excluidos digitales e las poblaciones de menores ingresos, grupos sociales que depende mucho más del servicio personal en las oficinas. La ampliación de los canales de atención bancaria por internet, no sirve para toda la población, por tanto las redes de oficinas bancarias siguen siendo fundamentales para el funcionamiento y ofrecimiento de servicios financieros, principalmente los grupos sociales más vulnerables.

Por último, señalar que se aprecia una tendencia clara, tanto en la ciudad de Lleida como en la de Presidente Prudente, siguiendo las lógicas de comportamiento de sus países, de incremento de servicios bancarios por medio redes digitales dirigidos al cliente, lo que refuerza aún más la trayectoria de disminución de las estructuras físicas y presenciales de atención bancaria, tendencia que produce cambios importantes en las prácticas cotidianas de los ciudadanos en el uso del tiempo y el espacio. Es el aprovechamiento de las redes digitales para la reconfiguración de la localización de los servicios financieros. 


\section{BIBLIOGRAFÍA}

ALONSO, M. P.; PUEYO, À.; POSTIGO, R.; LÓPEZ, C.; RUBIO, J. L. (a). La reestuctucturación del sector financiero, en: ALBERTOS, J. M. y SANCHEZ, J. L. (coords). Geografía de la crisis económica en España. Universidad de Valencia: JPM ediciones, 2014. Págs 305-328.

ALONSO, M. P.; PUEYO, À.; POSTIGO, R.; LÓPEZ, C. (b). Los efectos de la crisis financiera en la implantación territorial de los servicios bancarios: el caso de la ciudad de Zaragoza, en: ALBERTOS, J. M. y SANCHEZ, J. L. (coords). Geografía de la crisis económica en España. Universidad de Valencia: JPM ediciones, 2014. Págs 411-433.

BELLET S.,C. (Org.); MELAZZO, E.S. (Org.) ; SPOSITO, M.E.B. (Org.) ; LLOP, Josep M. (Org.) . Urganización, produción y consumo en ciudades medias/intermedias. 1. ed. Lleida: Universidad de Lleida, 2015. v. 1. 515p.

CARBÓ, S.. Mitos y realidades de la digitalización financiera. Los medios de pago como paradigma. Mediterráneo Económico, n²9, 2017, págs. 139-149.

CARROUÉ, L.. La planète financière: capital, pouvoirs, espace et territoires. Collection U, Géographie. Armand Colin. 2015. 250 Págs.

CASTELLS, M. La galáxia Internet - reflexions sobre internet, empresa y sociedad. Madri: Arete, 2001, p. 235 a 274.

CERNEV, A.; DINIZ, E.; JAYO, M.. Emergência da quinta onda de inovação bancária. Americas Conference on Information Systems (AMCIS), 2009

CONTEL, F. B.. Território e finanças. Técnicas, normas e topologias bancárias no Brasil. 1. ed. São Paulo: Annablume, 2011. v. 1. 316p.

DABAT, A.; HERNÁNDEZ, J., VEGA, C.. Capitalismo actual, crisis y cambio global. Economía UNAM, Vol. 12, nº 36., 2015, Pag. 62-89.

DIAS, L. C. Redes de informação, grandes organizações e ritmos de modernização. Etc (UFF), v. n.2, v1, p. 1-4, 2007.

DIAS, L. C.; LENZI, M. H.. Reorganização espacial de redes bancárias no Brasil: processos adaptativos e inovadores. Caderno CRH (UFBA. Impresso), v. 22, p. 97-117, 2009.

FEBRABAN. Pesquisa Febraban de Tecnologia Bancária. 2015. Disponível en: http://www.ciab.org.br/download/researches/ research-2015-en.pdf. Acesso: 02, fev de 2018.

FERNANDEZ, A. F. J.. La repercusión de la crisis en las entidades financieras: el caso de Lleida. Trabajo de fin grado. Departamento de Geografía y Sociología. Universitat de Lleida. 2014.

FERNANDEZ, J.C.. El riedling de la señora watababa. El impacto de la gran depresión en la geografía financiera de la deuda soberanana, en ALBERTOS, J.M. y SANHEZ, J.L. Geografía de la crisis económica en España. Universidad de Valencia, 2015, Págs. 77-99.

GARCÍA, F. J.. Aproximación crítica a la crisis económica mundial: Sistema capitalista, política monetaria y globalización financiera. Pecvnia, Revista de la Facultad de Ciencias Económicas y Empresariales. Universidad de León, nº10, 2010, págs.. 75-94.

GARROCHO, C.F y CAMPOS, J.. Organización espacial del sistema bancario dentro de la ciudad: estrategia territorial, accesibilidad y factores de localización. Economía, sociedad y territorio, n¹0 (33). 2010, págs. 413-453.

GARRIDO, A..Perpectivas del sistema bancario español: ¿evolución o revolución?. Mediterráneo Económico, n²9, 2017 , págs. 49-62.

HARVEY, D.. Condição Pós-Moderna. Uma pesquisa sobre as origens da mudança cultural. São Paulo: Loyola, 1993.

MATEACHE, P.. Las oficinas bancarias y el proceso de reestructuración en marcha. ATKearney, 2012.

MASSANELL, A. La transformación de la banca. Reorientación de los canales y servicios digitales. Papeles de Economía Española. Transformación digital en los medios de pago, $n^{\circ} 149.2016$, págs. 93-99.

MAUDOS, J.. Reestructuración bancaria y accesibilidad financiera. Cuadernos de información económica, enero/febrero, 2014.

MINOI, C., Atrapados en la red. Finanzas y desarrollo. Fondo Monetario Internacional, 49, n³, 2012, págs., 15-17.

OLIVEIRA, J. S.. Processos de inovação bancária recentes: ritmos de expansão e lógicas espaciais seletivas do setor bancário no município de Campinas - SP. In: Congresso Internacional Caleidoscópio da Cidade Contemporânea, 2, Campos dos Goytacazes: Edição Atual, 2017.

OLIVEIRA, J. S.. As lógicas espaciais do setor bancário nas cidades de São José do Rio Preto e Presidente Prudente: da estrutura espacial concentrada à multicentralidade seletiva. 2015.139 f. Monografia (Bacharelado em Geografia) - FCT/UNESP, Presidente Prudente. 2015.

ROJAS, L.. La revolución de las empresas FinTech y el futuro de la Banca. Disrupción tecnológica en el sector financiero. CAF. Banco de Desarrollo de América Latina, 2016, 43 págs. http://scioteca.caf.com/handle/123456789/976.

SANTOS, M.. Por uma outra globalização. Do pensamento único à consciência universal. São Paulo: Record, 2000. 
SCHERMA, R. A.. Topologias Bancárias no Período da Globalização. In: $1^{\circ}$ Simpósio de Pós-Graduação em Geografia do Estado de São Paulo, 2008, Rio Claro. Anais do 1 Simpósio de Pós-Graduação em Geografia do Estado de São Paulo, 2008.

SPOSITO, M. E. B.. Cidades médias: reestruturação das cidades e reestruturação urbana. In: SPOSITO, M. E. B.(Org.); et al. Cidades Médias: espaços em transição. São Paulo: Expressão Popular, 2007.

SPOSITO, M. E.. O chão em Presidente Prudente: a lógica de expansão territorial urbana. 1983. 230f. Dissertação (Mestrado em geografia) - UNESP Rio Claro. Rio Claro.

TROIANO, M.; SONDERGAARD, T.; THEODORE, S.. Multichannel and overcapacity: cost saving opportunity or competitive threat?. Scope Ratings, december, 2014.

VIEIRA, E.. Las crisis financieras de la globalización. Revista Civilizar Ciencias Sociales y Humanas, 14(26), 2014, 115-132. 